\title{
A Tabulation method for Character Recognition using Haar wavelet in 2D images
}

\author{
Chetan Shelke, Associate Professor, Department of Information Technology, Alliance University, \\ India, Chetan.shelke@alliance.edu.in
}

Mano Paul P, Associate Professor, Department of Information Technology, Alliance University, India, Chetan.shelke@alliance.edu.in

Ankita Nathe, Department of CSE, P. R. Pote (Patil) Education and welfare Trust's College of

Engineering \& Management, Amravati

Abstract: Character perception is a unique way to diagnostic the unidentifiable character into understandable text. Nowadays, with the increasing urge of getting information digital media has been roped in because of its convenient use. Hence the printed media needs to get into digital format but the printed text needs to get recognized by the computer this difficulty is solved by the pattern matching technique and haar wavelet method to recognize the character ,decompose it and frame it into understandable format keeping its prototype in mind this method can turn boon for the unidentifiable text also it can overcome the problems of printed text character perception system help human ease and reduce their jobs of manually handling and processing of document. The proposed method showed that the recognition of text is reliable and significantly accelerates data processing. Evaluation is executed as a collection of degraded documents which are images, indicates the proposed work will be well suited to reduce the noise by improving the pixel quality in which it will be rendered. The result analysis performed by using image quality matrix which are Mean Squared Error, Signal to Noise ratio, Structural Content, Normalized Cross Correlation, Maximum Difference, Normalize Absolute Error, Average difference. All the parameter shows effective results. Keywords - Character Perception, Haar Wavelet, Image Quality Matrix, Pattern Matching, Recognition of Text.

\section{INTRODUCTION}

In image processing, the most difficult tasks are recognition of text documents. Old documents and ancient texts have suffered from physical degradation of several types as a outcome of their age. The quality of many types of printed media, such as newspapers magazines and books, is also poor because of the often very thin or inferior papers used to produce them. Even modern books and texts which have not been kept in an appropriate environment may not be easy to read. As many of these physically damaged documents contain important information, efficient and accurate methods are needed to make that accurate and restore them. Problems in document images arise mainly from two sources: printing-imaging processes and physical phenomena. For report images which have endured debasement because of procedure surrenders, there are very well-created models which can be utilized to produce datasets of content as a solitary character, a solitary word, or even an entire page. A great part of the corruption endured by archive images that stems from physical procedures is the aftereffect of some sort of dissemination which happens over some undefined time frame. Such deformities are normal in extremely old archives, just as in media and reports made of low quality materials, and they are highly destructive [1].

These do not permit the application of conventional image recognition methods. Documents have been preserved in libraries around the world. To expose these documents safely in public domain such as libraries, it is important firstly to enhance their clarity, visibility and improve the quality. To remove the noise and increase image quality appropriate filtering methods need to be developed. Further, there is a need to apply appropriate image enhancement techniques, that is it used to enhance the image quality of these documents. Image Enhancement improves the clearness of input images for human looking, removes blurring and reduces noise and increases contrast for revealing more details. To maintain the original document persistently it is essential that these documents are transformed into digital form One of the objectives of proposed system is to preserve the historical documents forever [2]. 
Image processing techniques develop a digital system to analyze and manipulate the digital images for improvement in their quality. Image processing method gives an enhanced image and extracts useful information from it. These techniques process the historical images which are more legible for future reference and can be stored digitally. This may help to extend the life of the document. Thus, information will be preserved even if the physical document becomes completely obsolete and unreadable in the future [3].

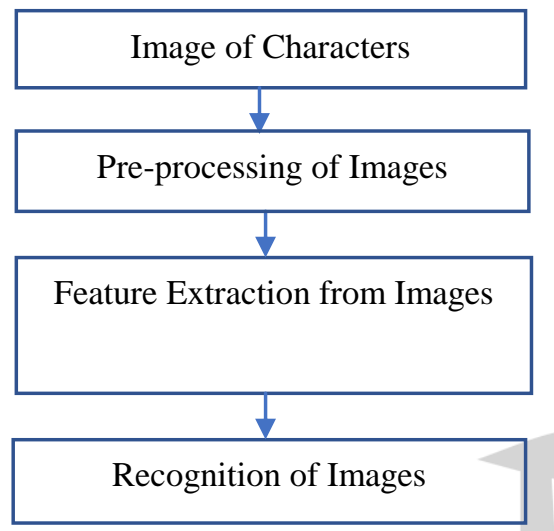

Figure 1 Components of Character Recognition

The above figure 1 shows components of Character Recognition System which are given below:-

Image of Character:- The image of character need to be selected to perform further stages to get character present in an image.

Pre-Processing:- The importance of the preprocessing stage of a character recognition method lies in its ability to remedy some of the problems Thus, the use of preprocessing techniques may enhance a document image setting it up for the following stage in a character acknowledgment framework[4]. So as to accomplish higher acknowledgment rates, it is fundamental to have a viable preprocessing stage, in this way; utilizing compelling preprocessing calculations makes the OCR framework progressively powerful essentially through exact picture improvement, commotion expulsion, picture thresholding, slant identification/remedy, page division, character division, character standardization and morphological strategies. Preprocessing strategies are required on shading, dim level or twofold archive pictures containing content as well as designs. In character acknowledgment frameworks the vast majority of the applications utilize dim or parallel pictures since handling shading pictures is computationally high. Such pictures may likewise contain non-uniform foundation and additionally watermarks making it hard to remove the archive content from the picture without playing out some sort of preprocessing. To accomplish the ideal come about because of preprocessing a few stages are required
- Some picture upgrade strategies to evacuate commotion or right the complexity in the picture

- Thresholding to evacuate the foundation containing any scenes, watermarks or potentially clamor

- Page division to isolate designs from content

- Character division to isolate characters from one another

- Morphological handling to improve the characters in situations where thresholding as well as other preprocessing methods dissolved pieces of the characters or added pixels to them.

The above procedures present not many of those which might be utilized in character acknowledgment frameworks and in certain applications; few or a portion of these methods or others might be utilized at various phases of the OCR framework. The rest of the chapter will present some of the techniques used during the preprocessing stage of a character recognition system [5].

Feature Extraction:- Features are the most relevant arrangement of qualities which characterize character pictures. Feature extraction in HCR is a significant field of picture handling and article acknowledgment. Major part of characters is called features. The fundamental undertaking of highlight extraction and choice is to discover a gathering of the best features for grouping; that is, compacting from high-dimensional element space to low-dimensional element space, in order to structure classifier successfully [6].

In factual methodology a character picture is spoken to utilizing a lot of $\mathrm{n}$ features which can be considered as a point in $\mathrm{n}$-dimensional element space. The primary objective of feature choice is to build direct or non-straight choice limits in include space that accurately independent the character pictures of various classes. Generally measurable methodology is utilized to diminish the element of list of capabilities for simple extraction and quick calculation where recreation of accurate unique picture isn't fundamental. These features are invariant to character distortion and composing style somewhat. A portion of the ordinarily utilized measurable features for character acknowledgment are projection histograms intersections, zoning and minutes and so on. The rapid improvement in technology makes character recognition system better in compatible cost. This is the cause of increasing the applications in different areas that can more effectively utilize. At times, there occur some circumstances where some documents are not in readable format or some specific part of it is doubtable, this proposed system helps to overcome this issue. This system also helps to interpret proper knowledge from varied sources and imparts a crystal clear understanding of the concerned document.

The aim of the proposed system is to develop a model to recognize English characters using Haar Wavelet 
decomposition, which is used for improving image quality and by using Template matching methodology for analyze shape and compare feature to match the input with resultant data. The basic characters included in English language are a set of numeral, a set of vowel, and a set of consonant. It is use to grow such an apparatus which accepts an symbol as info and concentrate characters (letter sets, digits, symbol) from it. The symbol can be of manually written report or Printed archive. It very well may be utilized as a type of information passage from printed records. Nature of the picture and goals of the picture are should be improved, and the exponential augmentation in calculation control is required to be made of new great calculation and its application.

The idea behind this system is dedicated to achieve some of the following objectives.

- To preserve the documents forever and the details of an image

- To Studying the various techniques used in recognizing of characters.

- To detect text regions present in the image.

- To retrieve text present in the image and display them to the user.

- To reconstruct original information and enhancement of low quality degraded images to higher frame quality.

- Analyzing the proposed recognition system with respect to the other recognition system obtained from other techniques.

\section{Literature SURVEY}

Numerous strategies have been proposed for character acknowledgment; they are frequently exposed to significant limitations because of sudden troubles. Verifiably character acknowledgment framework has developed in three ages to be specific the periods refered to noted in 1900-1980 (early ages) - The historical backdrop of character acknowledgment can be followed as ahead of schedule as 1900. At the point when the Russian Scientist Trying endeavored to build up a guide for outwardly impaired the primary character recognizers showed up in the center of 1940s with the improvement of computerized PCs. The past work on the programmed acknowledgment of characters has been thought either upon machine printed content or upon little arrangement of all around recognized written by hand content or images. The business character recognizers were accessible in 1950s [9].

In the Second ages 1980-1990 Developments - The examinations until 1980 experienced the tack of amazing PC equipment and information procurement infers. In any case, the character acknowledgment look into was centered around essentially the shape acknowledgment strategies without utilizing any semantic data.
After 1990 headways - The genuine advancement on character acknowledgment framework is accomplished during this period, utilizing the new procedures and improvement devices, which are enabled by constantly developing data advances. In the mid nineties, Image handling and Pattern acknowledgment procedures are proficiently joined with the Artificial Intelligence philosophies. Nowadays notwithstanding the more dominant PCs and precise electronic hardware's, for example, cameras, scanners and electronic tablets, we have effective utilization of philosophies, for example, Hidden Markov models, neural systems; Fuzzy set thinking and Natural language handling. Character acknowledgment framework is the base for a wide range of kinds of uses in different fields, which we use in our day by day lives.

Post Reclamation and Segmentation of Highly Degraded Characters Using a Shape-Independent Level Set Approach and Multi-level Classifiers is proposed by, R. F. Moghaddam, D. Rivest-Henault, and M. Cheriet in 2009 [11]. The method combines 2 approaches for segmentation. In first level, multilevel classifiers consider stroke width information to locate candidate character pixels. In second level, a set of active contour scheme is used to identify boundary of character. Stroke cavity map and estimation of intensity are considered in the method for segmenting degraded characters from degraded background. Tests are conducted on ancient degraded Hebraic character images. The methods are good for the images which have homogenous background images where we can see clear space between characters. However, this is not true for complex document images like Indus where we cannot expect regular spacing between characters.

Disconnected manually written English character acknowledgment dependent on convolutional neural system by A. Yuan, G. Bai, L. Jiao, and Y. Liu in 2012 [12]. This exploration applies Convolutional Neural Networks (CNNs) for disconnected manually written English character acknowledgment. We utilize a changed LeNet-5 CNN model, with unique settings of the quantity of neurons in each layer and the interfacing route between certain layers. Yields of the CNN are set with mistake rectifying codes, accordingly the CNN can dismiss acknowledgment results. For preparing of the CNN, a blunder tests based support learning system is created. For training of the $\mathrm{CNN}$, an error-samples-based reinforcement learning strategy is developed.

Text Line Detection in Corrupted and Damaged Historical Manuscripts is proposed by I. Rabaev, O. Biller, J. El-Sana, K. Kedem, and I. Dinstein in 2013 [13]. Technique gathered content lines by investigating the development maps of associated parts. A breadth line moved from left to right is additionally used to check whether components lie in a similar line. Be that as it may, 
the strategy can just identify lines of equivalent size writings which are picked in their dataset. Method is found to be powerful to detected characters in torn and damaged Manuscripts. The method extracts features which are invariant to degradations for text line segmentation. However, the method requires homogenous background images for achieving better results.

Text line extraction method in Scanned Document Gray Scale images was proposed by A. Asi, R. Saabni, and J. ElSana in 2014 [14]. The method is proposed directly on gray scale document images. In this paper another methodology for content line division that works straightforwardly on dark scale report pictures. Separation change is applied legitimately on the dark scale pictures, which is utilized to process two kinds of creases: Medial Seams and Separating Seams. A Medial Seam is a chain of pixels that crosses the content zone of a book line and a Separating Seam is a way that goes between two successive columns. The Medial crease decides a book line and the isolating creases characterize the upper and lower limits of the content line. The Medial and Separating Seams spread by vitality maps, which are characterized dependent on the developed separation change. Filtered Gray scale pictures have commotion. The separation change of boisterous record pictures may incorporate little vacillation that impacts Seam age. To defeat this impediment, Gaussian channels to smooth the picture before creating the separation change.

Penmanship picture upgrade utilizing neighborhood getting the hang of windowing, Gaussian Mixture Model and kimplies bunching by $\mathrm{H}$. Kusetogullari, H. Grahn, and N. Lavesson in 2016 [15]. Another methodology is proposed to upgrade the penmanship picture by utilizing learning-based windowing contrast improvement and Gaussian Mixture Model (GMM). A fixed size window moves over the penmanship picture and two quantitative techniques which are discrete entropy (DE) and edge-based complexity measure (EBCM) are utilized to gauge the nature of each fix. The got outcomes are utilized in the unaided learning technique by utilizing $\mathrm{k}$-implies bunching to allot the nature of penmanship as awful (in the event that it is low differentiation) or great (on the off chance that it is high complexity). From that point onward, if the comparing patch is assessed as low complexity, a differentiation upgrade strategy is applied to the window to improve the penmanship. GMM is utilized as a last advance to easily trade data among unique and upgraded pictures to dispose of the ancient rarities to speak to the last picture. BioInspired Modeling for the Enhancement of Historical Handwritten Documents by K. Zagoris and I. Pratikakis in 2017 [16].

Proposed system shows a significant advance for the record investigation and acknowledgment pipeline is the report picture binarization strategy. On account of authentic written by hand record pictures, the innate corruption of the archives requires a preprocessing step planning to upgrade the picture and improve the ensuing binarization step. To address this test another archive picture improvement strategy is proposed dependent on Bio-Inspired Models and particularly on the OFF-focus ganglion cells of the Human Vision System. Experimental results demonstrate the improvement of standard binarization methods when the proposed enhancement method is used.

Enhancement of Historical Documents by Image Processing Techniques is proposed by Neetu Mittal, Arjun Sehgal, Sunil Kumar Khatri in 2018 [17]. The proposed system aim to transform documents into digital form and enhance the quality and visualization of the historical images. Image processing techniques such as noise filtering, histogram equalization and power law transformation have been used to result in better representation of images. To evaluate the quality of resultant image, two performance assessment parameters; entropy and standard deviation, have been used.

\section{PROPOSED ANALYSIS AND DESIGN}

This system is designed to develop to recognize character present in an image file. Perceiving content in images is valuable in numerous PC vision applications, for example, picture search, archive examination, and robot route. The character acknowledgment work gives a simple method to add content acknowledgment usefulness to a wide scope of utilizations.

Character acknowledgment has gotten one of the best utilizations of innovation in the field of example acknowledgment and man-made reasoning. Surveillance systems have become applied to a number of different applications with Haar Wavelet decomposition, which is used for improving image quality and by using Template matching methodology for analyze shape and compare feature to match the input with resultant data. Different handy techniques for understanding this procedure has been formulated. Example coordinating strategy is one of the most regularly utilized procedures in which the similitude of info design is tried with the reference example of every classification. That project works under all possible conditions and gives highly accurate results still remains a highly challenging task to implement.

Documents suffer from several factors such as low paper quality, degradation, lack of standard alphabets, stains, noise, dense and arbitrary layout, typesetting imperfections, low print contrast and fonts etc. These do not permit the application of conventional image recognition methods to historical documents. Proposed concept addresses this.

In this system, feasibility means whether the techniques used for improving the quality for low quality degraded images is feasible to develop. In this work, feasibility study 
involves an examination of different techniques that are used for enhancing the image quality and detecting proper character. In this work, feasibility studies can be undertaken for all the methods and parameters that are taken as a part of all the phases for implementation and result evaluation. Here, different methods and parameters used for images enhancement for improving the character identification quality are describe, evaluated and finally selects the best system for development, that is called as Feasibility study. Three key considerations are involved in the feasibility analysis was also done namely Technical Feasibility, Economic Feasibility, Operational Feasibility.

\subsection{Requirement Analysis}

For performing our proposed system, that is for detecting text present in an image required character recognition mechanism. As the qualities of image are degraded it is difficult to detect any test from that image file. So we proposed to develop the technique for enhancing the frame quality by using Haar wavelet decomposition technique. The detailed mechanism and requirement study by identifying challenges is given below. The methodology and techniques used for developing makes the system more accurate and easy to handle, that makes this system more useful in all its application area.

Text Detection from image: Input image need to preprocess so as to remove the noise and enhance the features of image. The region of interest will be found by applying different algorithm to it and then register that image. then the pattern matching step in perform then ROI are matched with the expert images, if the infected image is not same then it will be added to the database and it matched then it shown the result.

At this point, all the detection results are composed of individual text characters. To use these results for recognition tasks, such as OCR, the individual text characters must be merged into words or text lines. This enables recognition of the actual words in an image, which carry more meaningful information than just the individual characters. For example, recognizing the string 'EXIT' vs. the set of individual characters $\left\{\mathrm{X}^{\prime}, \mathrm{E}^{\prime}, \mathrm{T}^{\prime}, \mathrm{I}^{\prime}\right\}$, where the meaning of the word is lost without the correct ordering.

Challenges Obtaining Accurate Results: This framework performs best when the content is situated on a uniform foundation and is arranged like a report. At the point when the content shows up on a non-uniform foundation, extra pre-handling steps are required to get the best outcomes.

\subsection{System Design}

System design contains diagrammatic representation of the proposed system. Where all the phases involved in character recognition system are explain in detail.
Image processing techniques develop a digital system to analyse and manipulate the digital images for improvement in their quality. Image processing method gives an enhanced image and extracts useful information from it. These techniques process historical images which are more legible for future reference and can be stored digitally. This may help to extend the life of the document. The proposed methodology provides a means for text preservation and image enhancement for poor quality and degraded document images. Main objective of this is to rendering the applications.

Their two main goals:

Preserve all the details of an image.

Absolute brightness information for tone mapped on an image provided in a low dynamic range.

To evaluate the quality of resultant image, performance assessment parameters; entropy standard deviation, Mean square error, Structural Content, Normalize Cross Correlation, Max Difference, Normalize Absolute Error, Average Difference have been used. The proposed method is much easier and may help to archive the documents in digital images with improved quality.

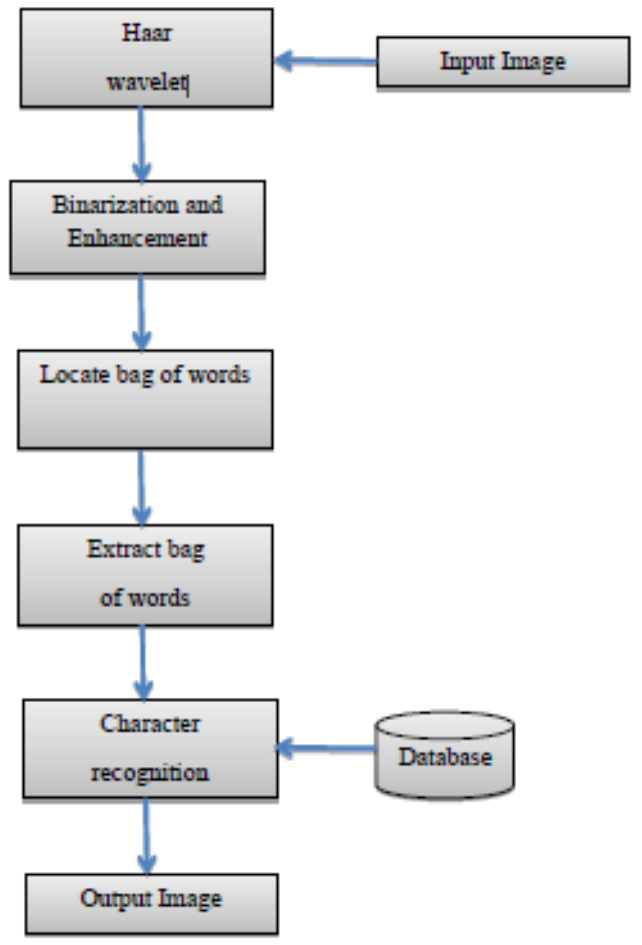

Figure 2 Proposed Design for Recognition

Image Acquisition:- First of all raw unprocessed image is provide as input, it can be any text that contain preferred language. Initially images are taken from internet. Which are later available in folder of the system that to apply the following procedures.

Image Decomposition: - The wavelet decomposition is nothing but to transform the original raw image into several 
components with single low-resolution component as shown in Figure 2 and 3. Figure 2 shows first level decomposition and figure 3 shows second level decomposition. A higher level of decomposition is obtained by repeating the same pattern of splitting it into number of rows and columns.

Enhancement takes the binary image matrix as input, examine it from top column to base line for each pixel of each line. In the event that it establishes any pixel in succession, at that point it accept it as the highest point of the picture. From that point forward, it again filters all pixel of each line and on the off chance that it establishes any pixel, at that point it will increase the line number. Toward the finish of the picture, it will spare the last line where it last found a dark pixel in the picture and accept that line as the base of the picture. Similarly, by the same process, it will detect the right and left the edge of that image. It will scan it from left most column to right most column for every pixel of each column. On the off chance that it finds any pixel in a section, at that point it accept it as the furthest left edge of the picture.

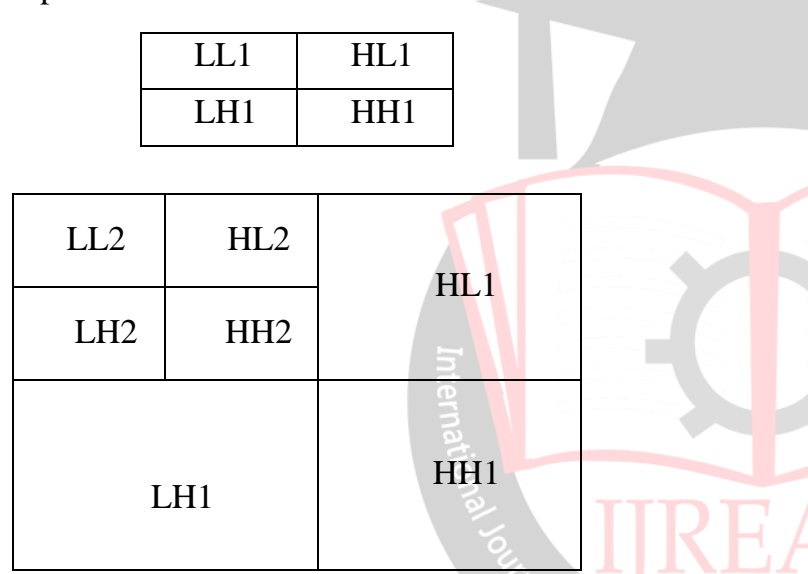

Figure 3 First level Decomposition

From that point onward, it again checks all pixel of each section and on the off chance that it finds any pixel it will increase the segment number. At the furthest right finish of a picture, it will spare the last segment number where it found the last dark pixel in the picture, and expect that segment as the furthest right edge of the picture.

Locate bag of words:- In the previous stage all images are decomposed. As character area are shown with white pixel and background with black. If character area of white pixel are greater than $90 \%$ then consider that there is character otherwise not.

Extract Image:- The left edge, right edge, top edge and bottom edge of the entire image are detected and cropped.

Character Reorganization:- The difficult stage is to recognize the character, here the matching operation is going to perform on the image which is provided as input and another one is the image from the dataset. Prior to coordinating, each picture from the dataset is taken and binarized. At that point both the gained information character picture and the binarized dataset picture is resized in a specific size. At that point it plays out a pixel by pixel coordinating activity between the info picture and all the put away picture in the dataset. It does likewise for all the dataset picture lastly, the one with the most extreme number of coordinated pixel is chosen and as needs be the proper character is identified from the information.

Character Printing: The character with the maximum value of the correlation coefficient is then printed on the screen in the form of an image.

Stepwise Workflow of the proposed system

Step 1: Input an image file

Step 2: Perform haar wavelet decomposition on an input image. Degraded frame are decomposed into number of frames of smaller by using Haar Wavelet decomposition technique. A higher level of decomposition is obtained by repeating the same pattern of splitting it into number of rows and columns.

Step 3: Image enhancement cotain binarization of image and also remove the unnecseeary background form the image Check the quality of the frame.

Step 4: Analyze image whether Bag of words found or not using Bag of words algorithm.

Step 5: Perform Binarization, remove noise present in an image and Enhancing quality of degraded frames by using enhance frame algorithm.

Step 6: Combining all the enhanced frames and making use of filter object algorithms for noise reduction.

Step 7: Combining all quality frames with enhanced frames and making use of template matching technique for detection and tracking of text in image

Step 8: Provide character recognize by system and Show results

\subsection{Proposed Methodology}

Haar Wavelet Transform

A wave is a fluctuating capacity of time or space and is occasional. Interestingly, wavelets are limited waves. Wavelet implies a "little waves". Wavelets are numerical apparatuses for stratified decaying capacities. Wavelets are mathematical functions which help in representing the original image into an image in frequency domain, which can else be divided into sub band images of different frequency components.

The Haar wavelet is a succession of rescaled "squaremolded" capacities which together structure a wavelet family or premise. The Haar arrangement was proposed in 1909 by Alfréd Haar.[1] Haar utilized these capacities to give a case of an orthonormal framework for the space of 
square-integrable capacities on the unit interval $[0,1]$.

One such type wavelet transform used here is Haar Wavelet Transformation. Haar wavelet enumerate a wavelet transform to represent image. It is the basic transformation from space to a local frequency domain. A HWT disintegrate each signal into two components, one is called average (approximation) or trend and the other is known as difference (detail) or fluctuation. This process is repeated repeatedly upto desired number levels by taking consideration of size of image /frame in the video.

\section{Properties of Haar Transform:}

Haar Transform is real and orthogonal.

The basis vectors of the Haar matrix are consecutively organized.

Orthogonally: The first sign is part into a low elimination round lattice $(\mathrm{T})$ whose lines and sections have a high recurrence part and channels empowering the veering without recreating data are said to symmetrical.

Linear Phase: To get straight stage, symmetric channels would need to be utilized. $\square$ Perfect reconstruction: If the input signal is transformed and inversely modified using a set of weighted basis functions and the reproduced sample values are equivalent to those of the input signal, the transform is said to have the perfect reconstruction property

\section{Haar Wavelet Decomposition Technique}

Wavelet transform sections the data of an image into approximation (low frequencies) and detail (high frequencies) sub-signals. The approximation (LL) subsignal shows the general trend of pixel values and other three detail sub-signals show the vertical (LH), horizontal (HL) and diagonal $(\mathrm{HH})$ details or changes in the images

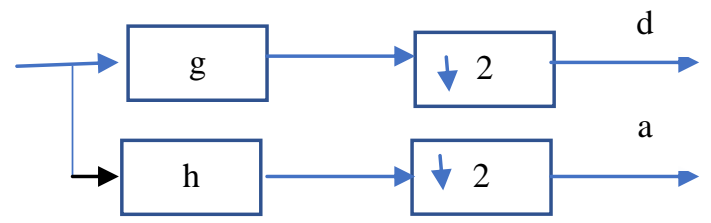

[18]. Splitting of signal into two parts shown in Figure 4.

Figure 4 Splitting of signals into two parts

\section{Template Matching Methodology}

Template matching is a powerful technique in digital image processing for finding small parts of an image which match a template image. This can also be used for classifying objects. Template matching techniques compare portions of images against one another. Sample image may be used to recognize similar objects in source image. Templates are most often used to identify printed characters, numbers, and other small, simple objects

Technique : Pattern matching
Operation : Analyze shape and compare features

Advantage: High Speed

Scope : Need huge data

Application: Image database available STEP 1:

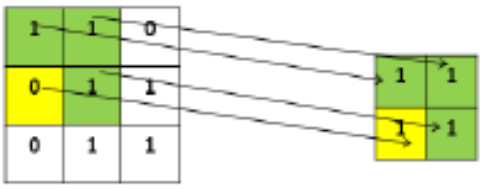

STEP 2:

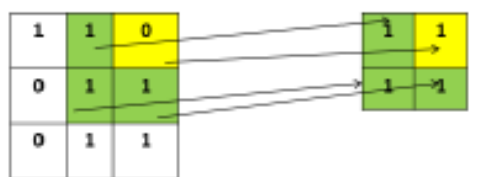

STEP 3:

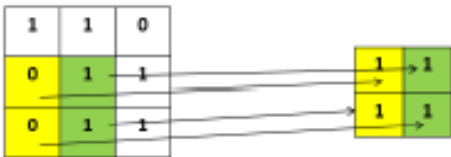

$\operatorname{STEP} 4$

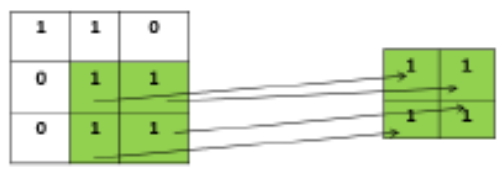

$-10096$ MATCHING

Figure 5 Template Matching Methodology

The detailed algorithms for character recognition are given below

Algorithm1: Haar Wavelet

Step 1: Start

Step 2: Input an Image I

Step 3: Input Decomposition Level L

Step 4: Resize an Image with size of Width \& Height as power of 2 .

Step 5: Initialize $\mathrm{i}=1$, Widthnew $=0$, Heightnew $=0$.

Step 6: While(1)

Widthnew_end $=$ Widthnew + Width $/$ L;

Heightnew_end= Heightnew + Height/L;

Di = Decompose an Image I (Widthnew, Widthnew_end ,

Heightnew, Heightnew_end)

Save Di

$\mathrm{i}=\mathrm{i}+1$;

Widthnew $=$ Widthnew_end;

Heightnew = Heightnew _end; End

Step 7: Save Result

Step 8: Stop

Algorithm 2: Extract Bag of Words

Step 1: Start

Step 2: Input Haar Decomposed Images database.

Step 3: For $i=1$ : length(Haar Database Hd) 
Read an Image I = Hd(i);

If I.contains (Char_Area) $>90 \%$

Crop an Image I

End

Step 4: Save Bag of Words.

Step 5: stop

Algorithm 3: Binarization

Step 1: Start

Step 2: Input an Image I;

Step 3: If $\mathrm{I}(;, ;, ;)>200$

Set I $(;, ;, ;)=255$

End

Step 4: Save Binary Image.

Step 5: Stop

Algorithm 3: Normalize Absolute Error

Step 1: Start

Step 2: Input an Image and

Step 3: Load database character

Step 4: For $\mathrm{i}=1$ to length (BOW)

For $\mathrm{i}=1$ to length (Database)

Find NAE (BOW, Database)

End

NAEindex $=$ Max $($ NAE $)$

Character $=$ NAE (index)

Display Character

End

Step 5: Save Result

Step 6: Stop

Where

BOW - Bag of words

NAE - Normalize Absolute Error

\section{EXPERIMENTAL RESUlTS}

Following screenshot shows complete execution of our proposed system:

1. This is the main window of our implemented model for our proposed work. At the left hand side it has all the buttons having functionality to be perform one after another in serial manner. The detailed description of this activity as input image, haar decomposition, image enhancement, locate bag of words etc. and all are briefly shown and explain in further screenshots.

Table 4.1 Analysis of different stages of Image with Measuring Parameter

\begin{tabular}{|l|l|l|}
\hline Parameter & Original Input Image & Enhance Image \\
\hline Entropy & 4.4987 & 0.15125 \\
\hline Mean of Image & 221.3125 & 253.5518 \\
\hline Standard Deviation & 61.1762 & 17.0107 \\
\hline Pure Height & 256 & 651 \\
\hline Pure Width & 768 & 4098 \\
\hline
\end{tabular}

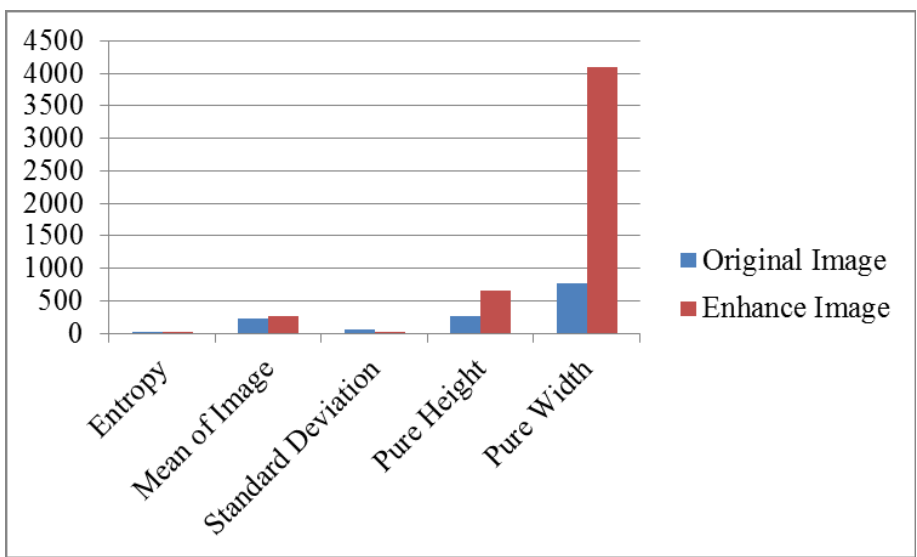

Figure 6 Comparison of Original image with Haar wavelet

In image enhancement technique quality of an image can be subjective. It can be different for person to person, so because of this reason it is compulsory to establish some parameters to get the quality of an image. Following are the Image Quality Metrics used to inquire the quality of images such as Mean Squared Error, Peak Signal to Noise ratio, Structural Content, Normalized Cross Correlation, Maximum Difference, Normalize Absolute Error, Average difference.

\section{Mean Squared Error}

MSE is a very simple and common distortion measure. MSE between the reference image and processed image with a size of a ( $\mathrm{mx} \mathrm{n})$ is expressed as follows:

MSE $=(1 / \mathrm{mn}) \Sigma \mathrm{m}-10 \Sigma \mathrm{n}-10 \mathrm{II} f(\mathrm{i}, \mathrm{j})-\mathrm{g}(\mathrm{I}, \mathrm{j}) \mathrm{II} 2$

Where $\mathrm{f}=$ matrix data of original image,

$\mathrm{g}=$ matrix data of degraded image,

$\mathrm{m}=$ no of rows of pixels of an image,

$\mathrm{i}=$ index of that row,

$\mathrm{n}=$ no of columns of pixels of an image,

$\mathrm{j}=$ index of that column.

where are the image pixel value of reference image and processed image respectively. The value of MSE measures the difference between a processed image and reference image. The smaller value of the MSE represents the better result.

Table 4.2 Image comparison of MSE

\begin{tabular}{|c|c|c|c|}
\hline $\begin{array}{c}\text { Image } \\
\text { Quality Metrics }\end{array}$ & $\begin{array}{l}\text { Input and } \\
\text { Binarize image }\end{array}$ & $\begin{array}{l}\text { Input and } \\
\text { BOW image }\end{array}$ & $\begin{array}{l}\text { Input and } \\
\text { Resultant image }\end{array}$ \\
\hline $\begin{array}{c}\text { Mean } \\
\text { Squared Error }\end{array}$ & 52560.2176 & $\begin{array}{lr} & 46055.378 \\
3 & \end{array}$ & 4567.4066 \\
\hline
\end{tabular}




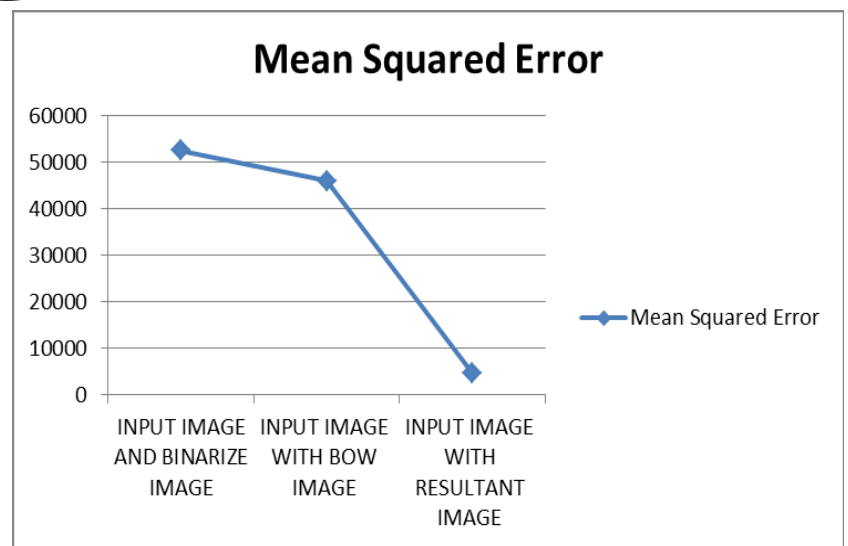

Figure 7 Comparison of Mean Square Error

\section{Peak Signal to Noise Ratio}

For the measurement of reconstruction quality, PSNR (Peak Signal to Noise Ratio) is one of the most extensively used metric parameter. It describes the ratio of the maximum possible power of a signal to the power of corrupting noise and is normally represented in decibel scale. PSNR can be expressed as follows:

PSNR $=10 \log 10($ R2 / MSE)

Where

$\mathrm{R}=255$ for an 8 -bit /class 8 image and $\mathrm{R}=1$ for $\mathrm{a}$ double precision image.

Table 4.3 Image comparison of PSNR

\begin{tabular}{|c|c|c|c|}
\hline $\begin{array}{c}\text { Image Quality } \\
\text { Metrics }\end{array}$ & $\begin{array}{c}\text { Input and } \\
\text { Binarize image }\end{array}$ & $\begin{array}{c}\text { Input and } \\
\text { BOW image }\end{array}$ & $\begin{array}{c}\text { Input and } \\
\text { Resultant } \\
\text { image }\end{array}$ \\
\hline $\begin{array}{c}\text { Peak Signal to } \\
\text { Noise Ratio }\end{array}$ & 0.92423 & 1.498 & 11.5341 \\
\hline
\end{tabular}

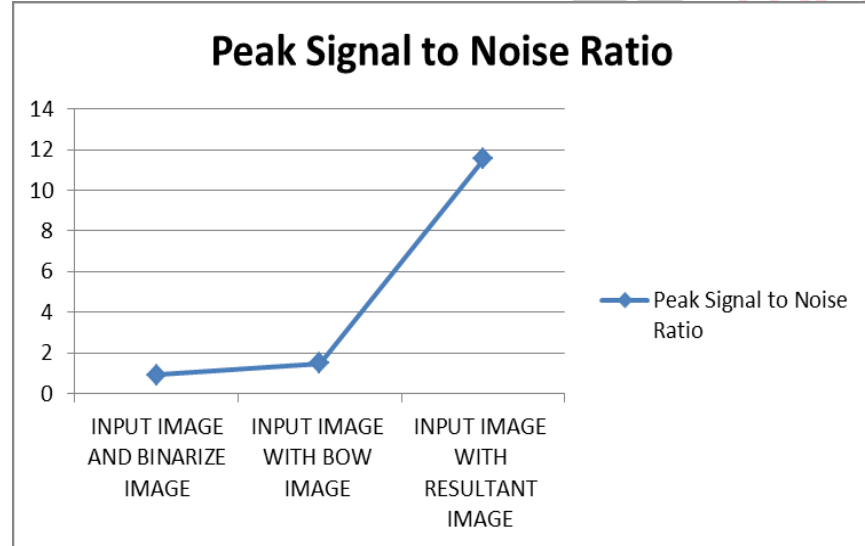

Figure 8 Comparison of Signal to Noise Ratio

Structural Content

This quality metric is expressed as follows:

$$
S C=\frac{\sum_{i=1}^{m} \sum_{j=1}^{n}\left(A_{j}\right)^{2}}{\sum_{i=1}^{n} \sum_{j=1}^{n}\left(B_{j}\right)^{2}}
$$

Table 4.4 Image comparison of SC

\begin{tabular}{|c|c|c|c|}
\hline $\begin{array}{c}\text { Image } \\
\text { Quality Metrics }\end{array}$ & $\begin{array}{c}\text { Input and } \\
\text { Binarize image }\end{array}$ & $\begin{array}{c}\text { Input and } \\
\text { BOW image }\end{array}$ & $\begin{array}{c}\text { Input and } \\
\text { Resultant image }\end{array}$ \\
\hline $\begin{array}{c}\text { Structural } \\
\text { Content }\end{array}$ & 287771.9023 & 4.2911 & 0.83715 \\
\hline
\end{tabular}

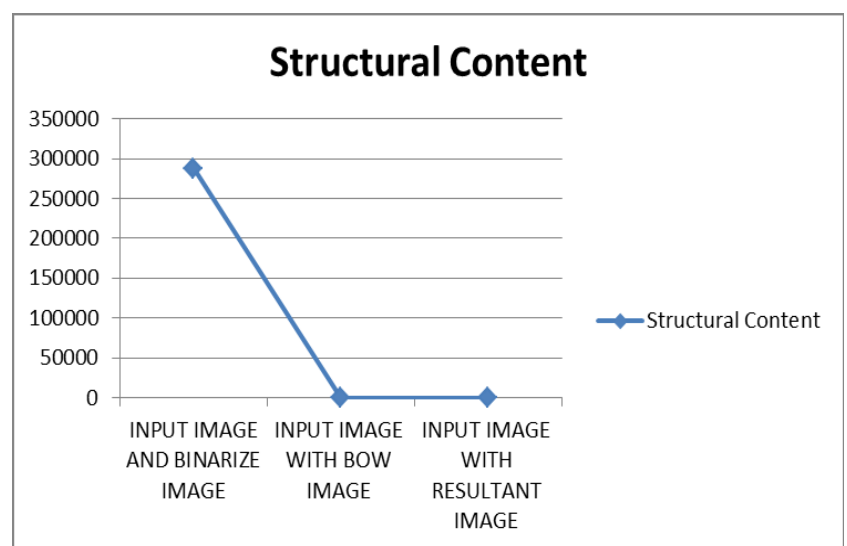

Figure 9 Comparison of Structural Content

Normalized Cross Correlation

NCC (Normalized Cross Correlation) measure shows the comparison of the processed image and reference image.

NCC is expressed as follows:

$$
N C C=\sum_{i=1}^{n} \sum_{i=1}^{n} \frac{\left(A_{i j} \times B_{y j}\right)}{A^{2} i j}
$$

Table 4.5 Image comparison of NCC

\begin{tabular}{|l|c|c|c|}
\hline $\begin{array}{c}\text { Image } \\
\text { Quality Metrics }\end{array}$ & $\begin{array}{c}\text { Input and } \\
\text { Binarize image }\end{array}$ & $\begin{array}{c}\text { Input and } \\
\text { BOW image }\end{array}$ & $\begin{array}{c}\text { Input and } \\
\text { Resultant image }\end{array}$ \\
\hline $\begin{array}{l}\text { Normalized } \\
\text { Cross } \\
\text { Correlation }\end{array}$ & 0.00035624 & 0.17871 & 1.0538 \\
\hline
\end{tabular}

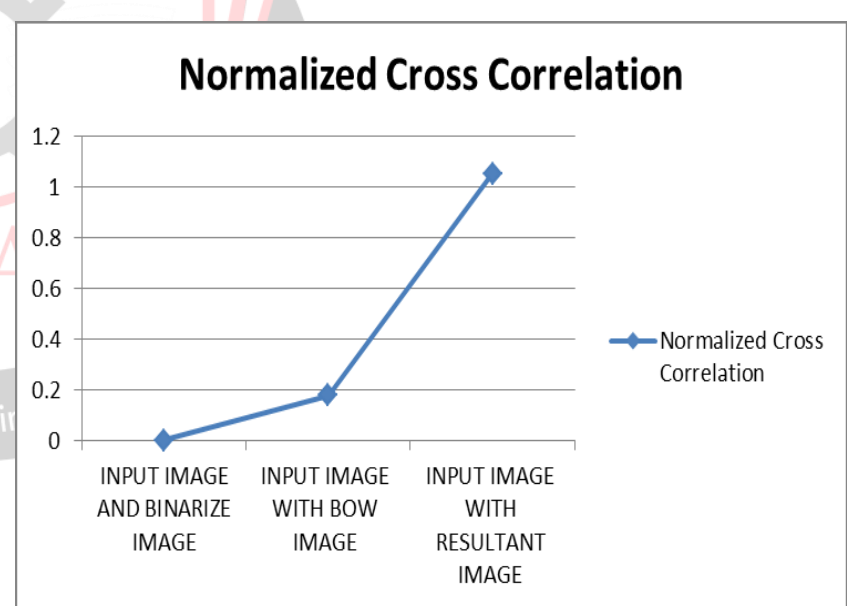

Figure 10 Comparison of Normalized Cross Correlation Maximum Difference

MD (Maximum Difference) provides the maximum of the error signal (i.e. difference between the processed and reference image). MD is defined as follows:

$$
\begin{aligned}
& \mathrm{MD}=\text { Max e|Aij }-\mathrm{Bij} \mid \mathrm{j} \ldots \ldots \ldots \ldots \\
& \mathrm{i}=1,2 \ldots \ldots \ldots \mathrm{m}, \mathrm{j}=1,2 \ldots \ldots \ldots \mathrm{n}
\end{aligned}
$$

Table 4.6 Image comparison of MD

\begin{tabular}{|c|c|c|c|}
\hline $\begin{array}{c}\text { Image } \\
\text { Quality } \\
\text { Metrics }\end{array}$ & $\begin{array}{c}\text { Input and } \\
\text { Binarize } \\
\text { image }\end{array}$ & $\begin{array}{c}\text { Input and } \\
\text { BOW image }\end{array}$ & $\begin{array}{c}\text { Input and } \\
\text { Resultant } \\
\text { image }\end{array}$ \\
\hline $\begin{array}{c}\text { Maximum } \\
\text { Difference }\end{array}$ & 255 & 255 & 164 \\
\hline
\end{tabular}




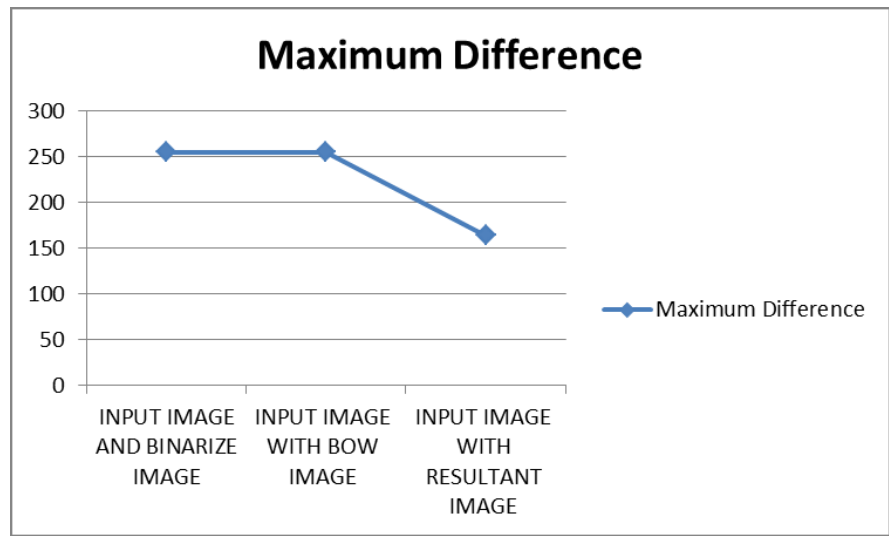

Figure 11 Comparison of Maximum Difference

The higher the value of the MD, the poorer the quality of the image.

Normalized Absolute Error

This quality measure can be expressed as follows. A higher NAE value shows that image is of poor quality.

$$
N A E=\frac{\sum_{i=1}^{m} \sum_{j=1}^{n}\left(\left|A_{i j}-B_{i j}\right|\right)}{\sum_{i=1}^{m} \sum_{j=1}^{n}\left(A_{i j}\right)}
$$

Table 4.7 Image comparison of NAE

\begin{tabular}{|l|c|c|c|}
\hline $\begin{array}{l}\text { Image } \\
\text { Quality } \\
\text { Metrics }\end{array}$ & $\begin{array}{c}\text { Input and } \\
\text { e Binarize } \\
\text { image }\end{array}$ & $\begin{array}{r}\text { Input and } \\
\text { BOW image }\end{array}$ & $\begin{array}{l}\text { Input and } \\
\text { Resultant } \\
\text { image }\end{array}$ \\
\hline $\begin{array}{l}\text { Normaliz } \\
\text { ed Absolute } \\
\text { Error }\end{array}$ & 0.99917 & 0.91125 & 0.15446 \\
\hline
\end{tabular}

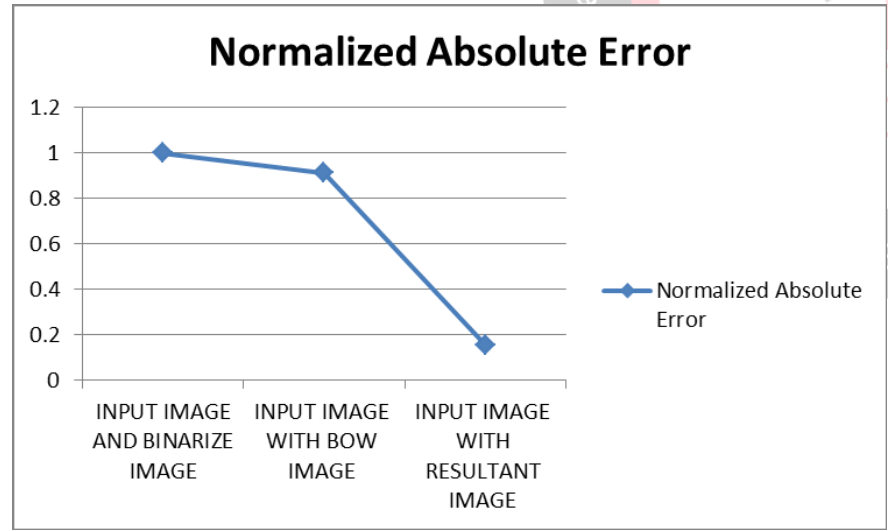

Figure 12 Comparison of Normalized Absolute Error Average Difference

$\mathrm{AD}$ (Average Difference) provides the average of change concerning the processed and reference image. AD can be expressed as follows:

$$
A D=\frac{1}{m n} \sum_{i=1}^{m} \sum_{i=1}^{n}[A(i, j)-B(i, j)]
$$

Table 4.8 Image comparison of AD

\begin{tabular}{|c|c|c|c|}
\hline $\begin{array}{c}\text { Image } \\
\text { Quality Metrics }\end{array}$ & $\begin{array}{c}\text { Input and } \\
\text { Binarize image }\end{array}$ & $\begin{array}{c}\text { Input and } \\
\text { BOW image }\end{array}$ & $\begin{array}{c}\text { Input and } \\
\text { Resultant } \\
\text { image }\end{array}$ \\
\hline $\begin{array}{c}\text { Average } \\
\text { Difference }\end{array}$ & 221.074 & 159.0699 & 28.5526 \\
\hline
\end{tabular}

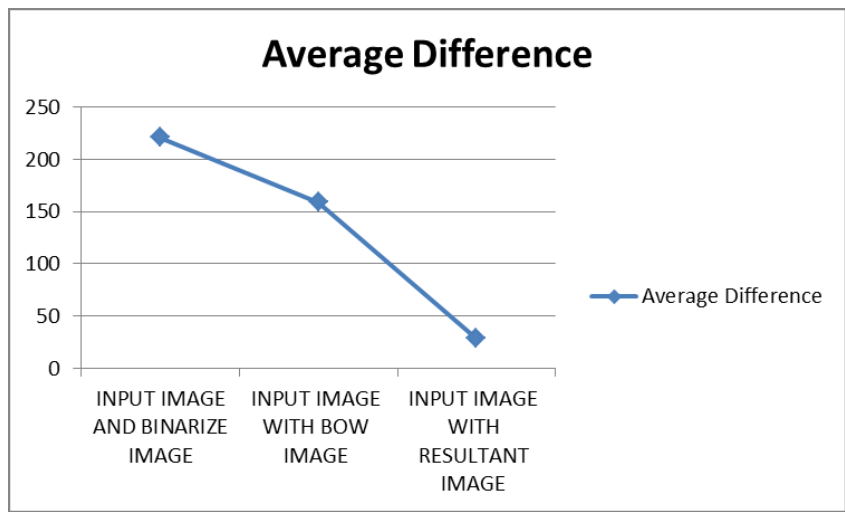

Figure 13 Comparison of Average Difference

\section{Conclusion}

An effective method for identification of characters was introduced that is Template matching with haar wavelet decomposition. It is the method used for character recognition techniques. Wavelets are mathematical functions which help in representing the original image into an image in frequency domain, which can else be divided into sub band images of different frequency components. The characters were extracted from input image and processes. For acknowledgment process, the extricated character was contrasted with every layout in the dataset to locate the nearest portrayal of the info character. Character acknowledgment is an ordering procedure of obscure info design into one of known limited number of character classes. Different reasonable strategies for understanding this procedure has been concocted. Example coordinating strategy is one of the most generally utilized procedures in which the closeness of info design is tried with the reference example of every classification. That project works under all possible conditions and gives highly accurate results still remains a highly challenging task to implement. This survey will be helpful for researchers in this field.

As far as the future directions of research presented in this thesis are concerned

Handwritten Character Recognition :- Handwriting recognition has been one of the active and challenging research areas in the field of image processing and pattern recognition The work can be extended for handwritten character recognition of any Indian languages

Online Character Recognition :- Online character recognition software is available on the Internet and is used directly online by people who need optical character recognition performed on files of various formats. An online character recognition service usually gives users the ability to convert more number of scanned images

\section{REFERENCES}

[1] N. Mittal, A. Sehgal, and S. K. Khatri, "Enhancement of historical documents by image processing techniques," 2017 6th International Conference on Reliability, Infocom 
Technologies and Optimization (Trends and Future Directions) (ICRITO), 2017.

[2] O. Boudraa, W. K. Hidouci, and D. Michelucci, "A robust multi stage technique for image binarization of degraded historical documents," 2017 5th International Conference on Electrical Engineering - Boumerdes (ICEE-B), 2017.

[3] K. Auliasari and M. Orisa, "Improving visual presentation of bas relief image from historical temple," 2018 International Conference on Information and Communications Technology (ICOIACT), 2018.

[4] Prabhdeep S and Arora A, "Analytical analysis of image filtering techniques", International Journal of Engineering and Innovative Technology (IJEIT), vol. 3, no.4, pp.234237,2013 .

[5] Shakair K and Mahmud J., "Salt and pepper noise detection and removal by tolerance based selective arithmetic mean filtering technique for image restoration", vol. 8, no. 6, pp.1234-1239, 2008.

[6] Mr. Chetan J. Shelke and Dr. Pravin Karde, "Traffic Sign Recognition", International Journal Of Computational Engineering Research (ijceronline.com) Vol. 2 Issue. 8, December 2012

[7] P. Janani, J. Premaladha and K. and S. Ravichandran, "noise removal techniques: A review", Indian Journal of Science and Technology, vol 8, No. 22, pp. 1-5, 2015.

[8] Papiya C, "Histogram equalization by cumulative frequency distribution", International Journal of Scientific and Research Publications. vol. 2, no. 7, pp.1-4, 2012.

[9] Dr. Jangala. Sasi Kiran , N. Vijaya Kumar , N. Sashi Prabha , M. Kavya4, "A Literature Survey on Digital Image Processing Techniques in Character Recognition of Indian Languages, vol 6(3),2015.

[10] Richard G. Casey And Eric Lecolinet " A Survey Of Methods And Strategies In Character Segmentation”, IEEE Trans. On Pattern Analysis And Machine Intelligence, Vol 18, Pp 690-706,1996.

[11] AR. F. Moghaddam, D. Rivest-Henault, and M. Cheriet, "Restoration and Segmentation of Highly Degraded Characters Using a Shape-Independent Level Set Approach and Multi-level Classifiers," 2009 10th International Conference on Document Analysis and Recognition, 2009.

[12] A. Yuan, G. Bai, L. Jiao, and Y. Liu, “Offline handwritten English character recognition based on convolutional neural network," 2012 10th IAPR International Workshop on Document Analysis Systems, 2012.

[13] I. Rabaev, O. Biller, J. El-Sana, K. Kedem, and I. Dinstein, "Text Line Detection in Corrupted and Damaged Historical Manuscripts," 2013 12th International Conference on Document Analysis and Recognition, 2013.

[14] A. Asi, R. Saabni, and J. El-Sana, "Text line segmentation for gray scale historical document images," Proceedings of the 2014 Workshop on Historical Document Imaging and Processing - HIP 11, 2014. image enhancement using local learning windowing, Gaussian Mixture Model and k-means clustering,” 2016 IEEE International Symposium on Signal Processing and Information Technology (ISSPIT), 2016.

[16] K. Zagoris and I. Pratikakis, "Bio-Inspired Modeling for the Enhancement of Historical Handwritten Documents," 2017 14th IAPR International Conference on Document Analysis and Recognition (ICDAR), 2017.

[17] N. Mittal, A. Sehgal, and S. K. Khatri, "Enhancement of historical documents by image processing techniques," 2017 6th International Conference on Reliability, Infocom Technologies and Optimization (Trends and Future Directions) (ICRITO), 2017.

[18] Shim, S.O., Malik, A.S., and Choi, T.S., "Accurate Shape from Focus Based on Focus Adjustment in Optical Microscopy", Microscopy Research and Technique, Volume 72, No. 5, pp. 362-370, 2009.

[19] Boissenin, M., Wedekind, J., Selvan, A.N., Amavasai, B.P., Caparrelli, F., and Travis, J.R., "Computer Vision Methods for Optical Microscopes", Image and Vision Computing, Volume 25, No. 7, pp. 1107-1116, 2007.

[20] Malik, A.S., and Choi, T.S., "Application of Passive Techniques for Three Dimensional Cameras", IEEE Transactions on Consumer Electronics, Volume 53, No. 2, pp. $258-264,2007$.

[21] Malik, A.S., and Choi, T.S., "Comparison of Polymers: A New Application of Shape from Focus", IEEE Transactions on Systems, Man, and Cybernetics, Part- C, Applications and Reviews, Volume 39, No. 2,pp. 246-250, 2009.

[22] Ahmad, M.B., and Choi, T.S., "A Heuristic Approach for Finding Best Focused Shape", IEEE Transactions on Circuits and Systems for Video Technology, Volume 15, No. 4, pp 566-574, 2005.

[23] Nayar, S.K., and Nakagawa, Y., "Shape from Focus", IEEE Transactions on Pattern Analysis and Machine Intelligence, Volume 16, No. 8, pp. 824-831, 1994.

[24] Thelen, A., Frey, S., Hirsch, S., and Hering, P., "Improvements in Shape-from-Focus for Holographic Reconstructions with Regard to Focus Operators, Neighborhood-Size, and Height Value Interpolation", IEEE Transactions on Image Processing, Volume 18, No. 1, pp. 151-157, 2009.

[15] H. Kusetogullari, H. Grahn, and N. Lavesson, "Handwriting 